\title{
Review of: "Clinical features and prognostic factors in spinal meningioma surgery from a multicenter study"
}

Wenqing Jia

Potential competing interests: The author(s) declared that no potential competing interests exist.

1. It will be better if this article can give more information about the surgical results of ventral spinal meningioma.

2. It will be better if some cases of resection of complex meningiomas could be given, such as spinal meningiomas surrounding the spinal cord.

3. It will be better if more information about spinal meningiomas with higher Simpson grade could be provided. 